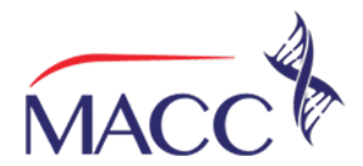

\title{
Interfascial Plane Blocks: Chasing The Unseen
}

\author{
Raden Besthadi Sukmono
}

Editor Majalah Anestesia \& Critical Care

U Itrasound has revolutionized anesthesia practice towards a better and safer technique. Regional anesthesia and pain medicine is one of the fields in anesthesiology that ultrasound has changed in so many ways. One of the main goals of regional anesthesia is to provide analgesia and immobility of certain parts of our body. In the early days of regional anesthesia, local anesthetics (LA) were injected blindly through a needle towards the biggest nerve responsible for innervating the surgical field. Anatomical landmarks based on the knowledge of surface anatomy were our only guiding light at the time. Also not to forget that little help from the uncomfortable tingling sensation experienced by our patients every time we came close to the neural target. This can be interpreted as success or danger, depending on how close the needle was from our target, an information that we did not have at that time. ${ }^{1}$

The 1980 s introduced us to nerve stimulator which successfully increased nerve identification with the use of electric current stimulating the motoric and sensory portion of the nerve. ${ }^{1,2}$ One of the earliest entry of ultrasound's application in regional anesthesia were in 1989, published in British Journal of Anaesthesia titled 'Ultrasonographic study of the spread of local anaesthetic during axillary brachial plexus block'. ${ }^{3,4}$ Since then, the era of ultrasound began with studies and publications spawning across various scientific journals. Regional Anesthesia and Pain Medicine published their first Ultrasound-guided regional anesthesia (UGRA) paper in 1992 titled 'Ultrasonic guidelines in lumbar sympathetic and celiac plexus block'.3,5 In the following couple of years, Anesthesia and Analgesia (AA) published their first UGRA paper titled 'Ultrasound-guided supraclavicular approach for regional anesthesia of the brachial plexus' ${ }^{3,6}$

As technology advances, especially in image quality department, identification of deeper and smaller structures has become possible. Studies that were initially made to describe the combination of landmarks and ultrasound images or to create new ultrasound markers, have since progressed to discuss the comparison with other tools or methods for nerve identification. We are now able to differentiate layers of neural structure (epineurium, perineurium, and endoneurium) with ultrasound image and manage to pinpoint the exact location for LA deposition. This translates as faster onset of blocks, enhancement of block quality, and safer blocks in terms of neurotoxicity and systemic toxicity. ${ }^{7}$ We are now brave enough to venture into deeper structures and implant perineural catheter. The refinements of our classic block process still continues until today. The costoclavicular approach ${ }^{8}$ and superior trunk approach for brachial plexus block ${ }^{9}$; individual blocks of axillary and suprascapular nerve for shoulder surgery ${ }^{10}$; and adductor canal block ${ }^{11}$ are to name but a few advances in the old blocks. Acute postoperative pain management, on the other hand, has become a problem that forced anesthesiologists to seek safer and more 
effective analgesia modalities. Opioids have always become the gold standards in this matter, although side effects ranging from respiratory depression, sedation, ileus, nausea and vomiting to pruritus limit their efficacy and utility. Regional anesthesia has long been employed as means of intraoperative and postoperative analgesia. The trunk or the chest and abdominal walls were the least considered sites to stick our block needle as they are mostly covered with neuraxial technique (spinal, epidural, and paravertebral). Neuraxial anesthesia is well established, but each carries serious side effects. Epidural block has high placement and catheter failure, urinary retention, and hemodynamic consequences that proves to be harmful especially to frail patients. Paravertebral block is an alternative for unilateral chest wall analgesia with less hemodynamic changes. Unfortunately, the landmark technique for paravertebral blocks were followed by reports of pneumothorax, failure, and unreliable block distribution with a single injection. Utilizing an ultrasound allows this block to be safer with better visualization of the pleura. Despite so, this technique still requires a certain level of expertise to master. ${ }^{12}$

The increasing popularity of minimally invasive surgery urges efforts to reduce surgical trauma and stress and consequently minimize postoperative pain from the surgeon's perspective. The concept that is also in line with the concept of Enhanced Recovery after Surgery (ERAS). These minimally invasive procedures are best be paired with anesthesia technique that are less potent than neuraxial blocks. Machi et al stated that this is the concept of minimally invasive regional anesthesia for acute postoperative pain. ${ }^{12}$

UGRA then delivered us transversus abdominis plane (TAP) block and it became the game changer in RA. This block is considered the originator of interfascial block. Not long after, the technique quickly evolved into a series of TAP block derivations (eg. subcostal TAP) and then followed by Pectoralis nerve blocks (Pecs) I, Pecs II, serratus anterior and quadratus lumborum (QL) blocks, transversalis fascia block, and the most recent and popular lately, erector spinae plane (ESP) block. ${ }^{13}$

Interfascial block works on a principle that by injecting local anesthetics in large volumes between two layers of fascia will create a potential space that bathe numerous neural targets residing in between these layers. This eventually block a certain area based on the extent of the space created. Multiple planar endpoints may exist for the same neural target due to the contiguous connection of fascial planes around the body. The fascia itself is soft connective tissue made of collagen, loose, and dense fibrous tissue, which surrounds organs, muscles, bones, and nerve fibers. Each fascia comprises multiple layers, and these layers represent the potential space for an interfascial block. The concept of interfascial block is entirely different than the traditional block where the neural target is visible. This concept made the exact target of the spread and indications of interfascial blocks are not always well defined. ${ }^{13}$ Take the QL block for instance. Quadratus lumborum muscle extends from the inferior border of the 12th rib until the iliac crest. The deposition of local anesthetics around this muscle will spread along with the extents of thoracolumbar fascia (TLF) produced sensory blocks ranging from T12 - L2 segment. Local anesthetics will also spread to paravertebral space and even covers the lumbar plexus. ${ }^{14,15}$ These facts that was proven through the dye spread in a cadaveric model made the indications of QL block widen to include analgesia for hip fracture surgery. ${ }^{16,17}$

The recent developments in interfascial blocks with numerous studies are of course not without any weakness. The most prominent issue concerning interfascial blocks is the inconsistency of block result. One day you could have the perfect block and not having it at all on the next day. One of the tricks to overcome this problem is to carefully watch the spread of local anesthetics in many ultrasound views after the initial injection. The perfect separation between layers is sometimes difficult to see, with most of the injectate going intramuscular. The other specific sign that was never reported in any publications is the 'breathing pattern'. This is the image viewed as the layers expand during injection and returns to a smaller state during aspiration or when the injection stopped. This cycle of volume expansion simulates chest wall 
movement during respiration, hence the name 'breathing pattern'. Unfortunately, this term is still considered a myth, since it is not backed by any studies to date. To facilitate the spread, the injectates are given in a quite large volume. Despite careful aspiration, vascular structure free images, and adjusted lower concentration of LA below calculated toxic dose, interfascial blocks still poses the risk of local anesthetic systemic toxicity (LAST). ${ }^{18}$

Application of interfascial plane block for upper extremity and chronic pain is not very common. Chronic and acute post-operative shoulder pain can be managed by applying interscalene block (ISB). Unfortunately, even with the high resolution images and low LA injectate volume, the incidence of hemi-diaphragmatic paralysis and phrenic nerve paralysis is not completely eliminated. This is particularly important in patients with severe pulmonary disease and obese individuals. ${ }^{19}$

The majority of the shoulder innervation is supplied distally by the axillary nerve and the suprascapular nerves, with minor contributions from subscapular and lateral pectoral nerves. The suprascapular nerve leaves the superior trunk close to the supraclavicular fossa and travels in the posterior triangle of the neck parallel to the inferior belly of the omohyoid muscle to enter the suprascapular notch. The subscapular nerves arising from the posterior cord are also present on the ventral surface of the subscapularis muscle. ${ }^{20}$ The lateral pectoral nerve is one of the nerve covered in pecs I interfascial block. ${ }^{21} \mathrm{An}$ inplane medial to-lateral approach of the needle is used to deposit $\pm 15 \mathrm{ml}$ of local anesthetic over the subscapularis muscle, which blocks both axillary and subscapular nerves by dispersion of the injectate along the ventral surface of subscapularis muscle. For the sub-omohyoid block, using an in-plane lateral to-medial needle approach, $\pm 5 \mathrm{ml}$ of the local anesthetic solution (ropivacaine $0.5 \%$ ) is deposited above the clavicle, under the inferior belly of omohyoid, to cover the suprascapular nerve. This fascial plane is located between the inferior belly of omohyoid and the strap muscles of the neck. ${ }^{19}$ Using the same technique aimed for postoperative pain management, Mubarak S wrote a case of successful procedural analgesia for awake frozen shoulder manipulation in an Indonesian female with frozen shoulder, ${ }^{22}$ a chronic painful shoulder condition. The dynamic pain score was 1 out of 10 throughout passive shoulder manipulation. Subsequently, the patient was able to actively move her upper arm. This report showed that interfascial block was successful in managing the pain for the simple but painful procedure. We are waiting for further studies regarding this technique in the future.

As an alternative for supplemental analgesia in the shoulder region, Kim DJ et al reported interfascial injection for axillary nerve block. The classical method is to identify posterior circumflex humeral artery (PCHA) and inject axillary nerve perivascular. In clinical practice, 2 or more vessels can be observed when the user is searching for the PCHA under ultrasound guidance, and the axillary nerve is often not sonographically visible. In addition, variation in the origin of the PCHA is common and it sometimes follows an abnormal course. Therefore, a new technique was developed to inject without the need for direct visualization of the PCHA or axillary nerve. The injectate placed in the interfascial space between the deltoid muscle and the teres minor muscle. The mean procedure time was significantly shorter in the interfascial group. There were no differences in the quality of blocks between the 2 groups. ${ }^{23}$

In the end, the knowledge of interconnecting potential space and multiple neural target opens the question that needed to be answered with studies and research about interfascial block. Are these minimally invasive blocks are as good as the real block? Will the clinical parameters and the future studies show that? We'll look forward to see them in the near future.

\section{REFERENCE}

1. Horlocker TT, Kopp SL, Wedel DJ. Peripheral Nerve Blocks. In: Miller RD, editors. Miller's Anesthesia. 8th Edition. San Diego: Churchil Livingstone; 2015. p1721-51.

2. Selander D, Edshage $S$, Wolff T. Paresthesiae or no paresthesiae? Nerve lesions after axillary blocks. Acta Anaesthesiol Scand. 1979; 23:27.

3. Neal JM, Brull R, Chan VWS, Grant SA, Horn 
JL, Liu SS, et al. The ASRA Evidence-Based Medicine Assessment of Ultrasound-Guided Regional Anesthesia and Pain Medicine Executive Summary. Reg Anesth Pain Med. 2010; 35: S1-S9

4. Ting PL, Sivagnanaratnam V. Ultrasonographic study of the spread of local anaesthetic during axillary brachial plexus block. $\mathrm{Br} \mathrm{J}$ Anaesth. 1989; 63:326-329.

5. Kirvela $O$, Svedstrom $E$, Lundbom $N$. Ultrasonic guidance of lumbar sympathetic and celiac plexus block: a new technique. Reg Anesth. 1992; 17: 43-46.

6. Kapral S, Krafft $P$, Eibenberger $K$, et al. Ultrasound-guided supraclavicular approach for regional anesthesia of the brachial plexus. Anesth Analg. 1994; 78: 507-513.

7. Antonakakis JG, Ting PH, Sites B. UltrasoundGuided Regional Anesthesia for Peripheral Nerve Blocks: An Evidence-Based Outcome Review. Anesthesiology Clin. 2011; 29: 179191.

8. Li JW, Songthamwat B, Samy W, SalaBlanch X, Karmakar MK. UltrasoundGuided Costoclavicular Brachial Plexus Block. Sonoanatomy, Technique, and Block Dynamics. Reg Anesth Pain Med. 2017; 42: 233-240

9. Burckett-St.Laurent D, Chan V, Chin KJ. Refining the ultrasound-guided interscalene brachial plexus block: the superior trunk approach. Can J Anesth. 2014 Dec; 61(12): 1098-102.

10. Price DJ. The shoulder block: a new alternative to interscalene brachial plexus blockade for the control of postoperative shoulder pain. Anaesth Intensive Care. 2007 Aug; 35(4): 575-81.

11. Jaeger $P$, Grevstad $U$, Henningsen $M H$, Gottschau B, Mathiesen O, Dahl JB. Effect of adductor-canal-blockade on established, severe post-operative pain after total knee arthroplasty: A randomised study. Acta Anaesthesiol Scand 2012;56:1013-9

12. Machi A, Joshi GP. Interfascial plane blocks. Best Practice \& Research Clinical Anaesthesiology. 2019; 33: 303-315

13. Elsharkawy $H$, Pawa A, Mariano ER. Interfascial Plane Blocks: Back to Basics. Reg Anesth Pain Med. 2018; 43: 341-346.
14. Blanco R, Ansari T, Girgis E. Quadratus lumborum block for postoperative pain after caesarean section. A randomised controlled trial. Eur J Anaesthesiol. 2015; 32:812-818.

15. Elsharkawy $\mathrm{H}$, El-Boghdadly $\mathrm{K}$, Barrington M. Quadratus Lumborum Block. Anatomical Concepts, Mechanisms, and Techniques. Anesthesiology. 2019; 130:322-35

16. Adhikary SD, El-Boghdadly K, Nasralah Z, Sarwani N, Nixon AM, Chin KJ. A radiologic and anatomic assessment of injectate spread following transmuscular quadratus lumborum block in cadavers. Anaesthesia. 2017; 72: 73-79

17. Kukreja $P$, MacBeth L, Sturdivant A, Morgan $\mathrm{CJ}$, Ghanem E, Kalagara $\mathrm{H}$, et al. Anterior quadratus lumborum block analgesia for total hip arthroplasty: a randomized, controlled study. Reg Anesth Pain Med. 2019; 44: 1075-1079

18. el-Boghdadly K, Pawa A, Chin KJ. Local anesthetic systemic toxicity: current perspectives. Local and Regional Anesthesia. 2018; 11: 35-44.

19. Sondekoppam RV, Lopera-Velasquez L-M, Naik L, Ganapathy S. Subscapularis and sub-omohyoid plane blocks: an alternative to peripheral nerve blocks for shoulder analgesia. $\mathrm{Br} J$ Anaesth. $2016 \mathrm{Dec}$; 117(6):831-832

20. Aszmann OC, Dellon AL, Birely BT, McFarland EG. Innervation of the human shoulder joint and its implications for surgery. Clin Orthop Relat Res. 1996; 330: 202-7.

21. Blanco R. The 'pecs block': A novel technique for providing analgesia after breast surgery. Anaesthesia. 2011; 66: 847-8.

22. Mubarak S. Shoulder Interfascial Plane Block as Sole Anesthesia for Frozen Shoulder Manipulation. MajAnestCriCar. 2020; 38 (3):185-7.

23. Kim ED, Baek JW, Kim JS, Oh SA, Kim YH. Ultrasound-Guided Block of the Axillary Nerve: A Prospective, Randomized, SingleBlind Study Comparing Interfascial and Perivascular Injections Pain Physician. July/ August 2019; 22: 369-375. 